\title{
Nonstationary System Analysis Methods for Underwater Acoustic Communications
}

\author{
Nicolas F. Josso, ${ }^{1}$ Jun Jason Zhang, ${ }^{2}$ Antonia Papandreou-Suppappola, ${ }^{2}$ Cornel Ioana, ${ }^{1}$ \\ and Tolga M. Duman ${ }^{2}$ \\ ${ }^{1}$ GIPSA-Lab/DIS, Grenoble Institute of Technology (GIT), 38402 Grenoble, France \\ ${ }^{2}$ School of Electrical, Computer and Energy Engineering, Arizona State University, Tempe, AZ 85287-9309, USA
}

Correspondence should be addressed to Antonia Papandreou-Suppappola, papandreou@asu.edu

Received 3 August 2010; Accepted 26 December 2010

Academic Editor: Antonio Napolitano

Copyright () 2011 Nicolas F. Josso et al. This is an open access article distributed under the Creative Commons Attribution License, which permits unrestricted use, distribution, and reproduction in any medium, provided the original work is properly cited.

\begin{abstract}
The underwater environment can be considered a system with time-varying impulse response, causing time-dependent spectral changes to a transmitted acoustic signal. This is the result of the interaction of the signal with the water column and ocean boundaries or the presence of fast moving object scatterers in the ocean. In underwater acoustic communications using mediumto-high frequencies $(0.3-20 \mathrm{kHz})$, the nonstationary transformation on the transmitted signals can be modeled as multiple timedelay and Doppler-scaling paths. When estimating the channel, a higher processing performance is thus expected if the techniques used employ a matched channel model compared to those that only compensate for wideband effects. Following a matched linear time-varying wideband system representation, we propose two different methods for estimating the underwater acoustic communication environment. The first method follows a canonical time-scale channel model and is based on estimating the coefficients of the discrete wideband spreading function. The second method follows a ray system model and is based on extracting time-scale features for different ray paths using the matching pursuit decomposition algorithm. Both methods are validated and compared using communication data from actual underwater acoustic communication experiments.
\end{abstract}

\section{Introduction}

Most physical systems can be represented by models that account for the transformations caused on the propagating signal. Depending on these transformations, linear timevarying (LTV) systems have been represented using narrowband, wideband; or dispersive nonstationary models [1-3]. Although all LTV systems can be characterized by a kernel representation of their time-varying impulse response $[4,5]$, they can also be identified by a matched spreading function that can provide a physical interpretation of the system effects on the propagating signal [5-8]. For example, a typical wireless communication system utilizing electromagnetic waves over the air can be considered to be a narrowband LTV system undergoing time shifts (due to multipath propagation and time dispersion) and frequency shifts (due to relative motion between transmitters and receivers) [5, 7, 9-11]. The received signal can be described as a superposition of time and frequency shifted replicas, weighted by the narrowband spreading function. Thus, estimating the narrowband spreading function can provide a means for improving communication receiver performance $[11,12]$.

Characterizing acoustic signal propagation through water is essential for many applications, including underwater acoustic communications, active and passive sonar, underwater navigation and tracking, and ocean acoustic tomography. The highly time-varying nature of the underwater environment can cause many undesirable distortions to the propagating signal. Time-varying multipath distortions may be the result of dense reflections from rough surfaces, fluctuations in sound speed due to inhomogeneous mediums, relative motion between transmitters and receivers, or changes in the propagating medium [13]. Depending on the transmission frequency and ocean depth, the time-dependent spectral changes in the signal can be Doppler scaling (compression or expansion) or dispersive (nonlinear) transformations $[3,6,14]$. In particular, medium-to-high frequency $(0.3-20 \mathrm{kHz})$ underwater acoustic signals are characterized by spreading caused by multiple time-delay paths and multiple Doppler-scaling 
paths [15-17]. As the narrowband LTV model is no longer suitable to describe these signal transformations, the matched wideband LTV model should be used for more effective processing [1, 8, 18-22].

Underwater acoustic signals were characterized using different techniques in the literature. Specifically, signal characteristics were extracted to evaluate underwater multipath profiles for use in shallow-water localization and geoacoustic inversion applications in [23]. In [24], inverse problems with matched filtering were considered in underwater acoustics. Specifically, when the motion of the transmitter and receiver, and the changes in the propagating medium were assumed not known, the received signal was correlated with a family of reference signals representing all possible transmissions. The Dopplerlet transform was used to estimate the range and speed of a moving source in [25]. In [17], a long range multipath profile estimation method was used based on the wideband ambiguity plane [26] that resampled the received signal with the dominant Doppler scale factor. Other recent multipath profile estimation methods considered the joint estimation of multiple time delays and Doppler scales [27, 28].

Different processing techniques were developed to estimate the parameters of fast varying communication channels that compensated for the wideband effect instead of actually using a model that matched the channel. In [29-31], the underwater acoustic channel was assumed to have the same Doppler scale on all propagation paths so that the estimated Doppler scaling could be mitigated by resampling the received signal. Although the channel was modeled with multiple Doppler scale paths in $[15,32,33]$, the channel estimation approach still assumed a single dominant Doppler scale and compensated for the residual Doppler in the different arrival paths by assuming different frequency shifts. In [34], Doppler scale was first compensated for using a mean scale factor before assuming a narrowband timefrequency spreading representation of the received signal and using the matching pursuit decomposition algorithm to sequentially identify dominant taps of sparse underwater acoustic communication channels and estimating their coefficients. Note, however, that although different frequency shifts were considered for different time delays, the narrowband model is not valid when the bandwidth-tocentral-frequency ratio becomes larger than 0.1 [18], as is the case for typical orthogonal frequency-division multiplexing (OFDM) communication signals.

In this paper, we propose two methods for estimating the parameters of underwater acoustic communication channels that characterize signals with multiple time-delay and Doppler-scaling path propagations. As such, they can be used to improve the performance of communication channels over existing underwater acoustic processing algorithms. Specifically, we directly use the wideband LTV system representation $[1,18,19]$ since the underwater acoustic environment can exhibit large multipath spreading and Doppler-scale spreading effects. Using this representation, the communication channel is characterized by a continuously varying wideband spreading function (WSF) that can directly describe the physical effect of the channel's intensity and spread on the transmitted signal.
The first proposed characterization method estimates the coefficients of the smoothed and sampled WSF based on a discrete version of the wideband LTV system representation. The discrete canonical model was initially proposed to improve efficiency in processing and provide improved performance using model-inherent diversity paths $[3,8,20$, 21]. The model decomposes the received signal into a linear combination of time-shifted and Doppler-scaled versions of the transmitted signal, weighted by a smoothed and sampled version of the WSF. The second proposed characterization method is specifically applied to communication channels that can be represented using the ray theory model. According to this model, the transmitted signal undergoes only a small number of multipath and Doppler scale changes. Thus, instead of directly estimating the WSF, we employ the wideband ambiguity function and the matching pursuit decomposition (MPD) [35] algorithm, with well-matched wideband basis functions, to estimate the wideband channel attribute parameters.

The rest of the paper is organized as follows. In Section 2, we provide the discrete wideband LTV channel model formulation in terms of the smoothed and sampled WSF. We also provide a least-squares estimation method for estimating the WSF coefficients together with a more computationally efficient method for realistic communication channels based on warping and time-frequency filtering techniques. An MPD-based method for estimating the characteristics of sparse underwater acoustic channels following the ray theory model is provided in Section 3. Sections 4 and 5 present our channel estimation results using two sets of real experimental data.

\section{Discrete Time-Scale Channel Characterization}

2.1. Wideband Nonstationary Model. Most underwater acoustic communication signals are considered to have wideband properties due to the movement of scatterers in the channel causing Doppler-scaling signal transformations. In many cases, the wideband Doppler-scaling effect can be approximated by frequency shifts. However, this narrowband approximation only holds when underwater scatterers move slowly and when the transmitted signal bandwidth is much smaller than its central frequency. As wideband underwater acoustic signals with spectral components in the 300 to $20,000 \mathrm{~Hz}$ frequency range have bandwidths that are comparable to their central frequencies, they are characterized by time-delay and Doppler-scale changes.

The wideband LTV channel model represents the channel output in terms of continuous time-delay and Doppler-scale change transformations on the transmitted signal, weighted by the WSF. Specifically, the noiseless received signal $x(t)$ can be represented as $[1,3,8,18-20]$

$$
x(t)=\int_{0}^{T_{\text {delay }}} \int_{\eta_{\min }}^{\eta_{\max }} \mathcal{X}(\tau, \eta) \sqrt{\eta} s(\eta(t-\tau)) d \eta d \tau,
$$

where $\tau$ and $\eta$ are the continuous time-delay and Dopplerscale parameters, respectively, and $s(t)$ is the transmitted 
signal. The WSF, $\mathcal{X}(\tau, \eta)$, represents the random phase change and attenuation of underwater scatterers corresponding to different values of $\tau$ and $\eta$. Due to path loss or velocity limit restrictions of realistic underwater acoustic channels, we assume that the WSF support regions are $\tau \in\left[0, T_{\text {delay }}\right]$ and $\eta \in\left[\eta_{\min }, \eta_{\max }\right]$, where $T_{\text {delay }}$ is the channel's time-delay spread, and the range of possible scaling values is given by $\left[\eta_{\min }, \eta_{\max }\right]$.

In $[8,20]$, we derived a discrete version of the timescale representation in (1) for use in real-time processing. We obtained the discrete formulation by geometrically sampling the scaling parameters using the Mellin transform $[20,36]$ and by uniformly sampling the time-delay parameters. The discrete time-scale representation is given by [20]

$$
x(t)=\sum_{m=M_{0}}^{M_{1}} \sum_{n=0}^{N(m)} \Psi_{n, m} \eta_{0}^{m / 2} s\left(\eta_{0}^{m} t-\frac{n}{W}\right),
$$

where $\Psi_{n, m}$ are smoothed and sampled versions of the WSF coefficients, $M_{0}=\ln \left(\eta_{\min }\right) / \ln \left(\eta_{0}\right), M_{1}=\ln \left(\eta_{\max }\right) / \ln \left(\eta_{0}\right)$, $N(m)=\eta_{0}^{m} W T_{\text {delay, }}$, and $m$ is an integer. Here, $W$ is the frequency-domain bandwidth of $s(t), \eta_{0}=e^{1 / \beta_{0}}$ and $\beta_{0}$ is the Mellin-domain support of $s(t)$. Thus, the time-delay $\tau=n /\left(\eta_{0}^{m} W\right), n=0,1, \ldots, N(m)$, is uniformly sampled for each given scaling factor $\eta_{0}^{m}$. Note that the number of time-delay parameters is not the same for all scaling factors since it is a function of the scale factor $m$ in (2). Specifically, the number of canonical time-scale components is $\mathcal{N}=$ $\sum_{m=M_{0}}^{M_{1}}(N(m)+1)$. In realistic underwater environments, due to large multipath spreads, even if only a few scale factors are considered, the number of time-scale components can be large. For example, using nine scale factors and a channel with time-delay spread $T_{\text {delay }}=0.15 \mathrm{~s}$, the number of timescale paths is 3,000 .

2.2. Direct WSF Estimation. Using the discrete time-scale channel representation in (2), we can estimate the WSF coefficients $\Psi_{n, m}$ using a least-squares estimation approach. Specifically, we uniformly sample the received signal $x(t)$ and form the vector $\mathbf{x}$. We also uniformly sample the timeshifted and Doppler-scaled version of the transmitted signal, $\eta_{0}^{m / 2} s\left(\eta_{0}^{m} t-n / W\right)$, and form $\phi_{n, m}$. We concatenate $\phi_{n, m}$ to form the data matrix $\mathbf{D}=\left[\phi_{0, M_{0}} \phi_{1, M_{0}} \cdots\right.$ $\left.\phi_{N\left(M_{0}\right), M_{0}} \phi_{0, M_{0}+1} \cdots \phi_{N\left(M_{0}+1\right), M_{0}+1} \cdots \phi_{0, M_{1}} \cdots \phi_{N\left(M_{1}\right), M_{1}}\right]^{T}$, where $T$ denotes transpose. We similarly concatenate the coefficients $\Psi_{n, m}$ to form the WSF coefficient vector $\Psi=$ $\left[\Psi_{0, M_{0}} \Psi_{1, M_{0}} \cdots \Psi_{N\left(M_{0}\right), M_{0}} \cdots \Psi_{0, M_{1}} \cdots \Psi_{N\left(M_{1}\right), M_{1}}\right]^{T}$. The discrete time-scale system representation can then be rewritten in matrix form as $\mathbf{x}=\mathbf{D \Psi}$, and the WSF coefficients can be estimated using the least-squares estimation method to yield

$$
\hat{\Psi}=\left(\mathbf{D}^{T} \mathbf{D}\right)^{-1} \mathbf{D}^{T} \mathbf{x}
$$

Note that $\mathbf{D}$ can be formed from a dictionary containing all possible time-delay and Doppler-scale transformations on the transmitted signal.
2.3. WSF Estimation with Reduced Computational Complexity. In realistic scenarios, the propagating paths have been observed to arrive in groups of similar time-delay and Doppler-scale components due to physical constraints in the propagation medium $[17,26]$. Hence, in order to reduce the computational complexity in estimating the WSF at the receiver, we detect and separate each major path group by first applying a warping based filtering technique in the wideband ambiguity function (WAF) lag-Doppler plane and then by estimating the WSF coefficients corresponding to each path group using a least-squares approach.

The warping lag-Doppler filtering (WALF) approach aims to provide an efficient way of separating the different path groups in the WAF plane. We start by computing the WAF of the received signal using a dictionary of time-shifted and scaled versions of the transmitted signal. Given the transmitted signal $s(t)$, we define a signal dictionary $\mathscr{D}$ that consists of all possible signals received after propagating over the wideband channel, as in (1). These signals in $\mathscr{D}$ are given by

$$
g^{(m, n)}(t)=\sqrt{\left|\eta_{m}\right|} s\left(\eta_{m}\left(t-\tau_{n}\right)\right), \quad \eta_{m} \neq 0,
$$

with all possible $\tau_{n}$ and $\eta_{m}$ chosen to represent the appropriate range of time delays and scale changes, respectively. The WAF of the received signal $x(t)$, defined over the same ranges, is given by

$$
R_{x}\left(\tau_{n}, \eta_{m}\right)=\left\langle x, g^{(m, n)}\right\rangle \triangleq \int_{-\infty}^{\infty} x(t) g^{(m, n) *}(t) d t .
$$

The steps of the WALF iterative algorithm are summarized as follows. We first initialize the algorithm by setting $x(t)=b_{0}(t)$. Then, at the $i$ th iteration, $i=0,1, \ldots, M-1$, we compute the projection $\Lambda_{i}^{(m, n)}$ of the residue $b_{i}(t)$ onto every dictionary element $g^{(m, n)}(t) \in \mathbb{D}$ as the WAF of the residue. That is, we obtain the projection as $\Lambda_{i}^{(m, n)}=$ $R_{b_{i}}\left(\tau_{n}, \eta_{m}\right)$ in (5). As a result, the local maxima of the WAF are reached for each path group. Specifically, for the $i$ th path group, the WAF reaches a local maxima when the reference and analyzed signals have a match in their time-delay and Doppler-scaling factors [17]. More precisely, the absolute maximum is reached when the reference signal matches the signal received for the most energetic propagation path of the corresponding path group. Hence, we select the dictionary signal $g_{i}^{\left(m_{i}, n_{i}\right)}(t)$, with time-shift $\tau_{n_{i}}$ and scale $\eta_{m_{i}}$, which maximizes the magnitude of the projection

$$
g_{i}^{\left(m_{i}, n_{i}\right)}(t)=\arg \max _{g^{(m, n)}(t) \in D}\left|\Lambda_{i}^{(m, n)}\right| .
$$

For realistic applications, we can assume that the derivative of the phase function $\varphi(t)$ of $s(t)$ exists and is positive. We also assume that $\varphi(t)$ is known as $s(t)$ is assumed known, and we let $\varphi_{i}(t)=\varphi\left(\eta_{m_{i}}\left(t-\tau_{n_{i}}\right)\right)$ represent the phase function of $g_{i}^{\left(m_{i}, n_{i}\right)}(t)$. As we need to separate each arrival path group, and the different arrival path groups will have different phase functions according to $\varphi_{i}(t)$, a different time-varying filtering approach needs to be applied in the WAF plane. Thus, due 
to their different nonstationary patterns, the different arrival path groups are not linearly separated in time frequency (TF). As a result, TF-based filtering cannot be used directly. Hence, we propose to use TF-based filtering that operates in the warped TF domain, where the path families can be separated $[28,37]$.

Warping is a method to nonlinearly map one domain onto a new domain, where processing can be more easily applied [38-41]. We use the linear and unitary warping operator $\mathcal{W}_{u}$ with associated warping function $u(t)$ that transforms a square-integrable signal $g(t) \in \mathcal{L}^{2}(\mathbb{R})$ as [39, 42]

$$
\left(\mathcal{W}_{u} g\right)(t)=\left|\frac{d u(t)}{d t}\right|^{1 / 2} g(u(t))
$$

We warp the residue $b_{i}(t)$ using the time warping operator $\mathcal{W}_{\varphi_{i}^{-1}}$ with $u(t)=\varphi_{i}^{-1}(t)$ in (7) to obtain

$$
Q_{i}(t)=\left(\mathcal{W}_{\varphi_{i}^{-1}} b_{i}\right)(t)
$$

where $\varphi_{i}\left(\varphi_{i}^{-1}(t)\right)=t$ for all $t$. Since $b_{i}(t)$ follows from (4) and (6), and the phase of $b_{i}(t)$ is $\varphi_{i}(t)$, then the warped signal $Q_{i}(t)$ in (8) is a sinusoid. As such, it can be filtered out easily in the warped time domain, as desired. According to the propagation properties, $\varphi_{j} \neq \varphi_{i}$, for all $j \neq i, j=0,1, \ldots, M-$ 1 . As a result, only the signal received for the $i$ th path group is filtered out using the passband filter $\delta$ to remove the narrowband function received for the $i$ th path group after the WALF operation $U_{i}(t)=\delta Q_{i}(t)$. The projection is unwarped in the time domain and the signal of the next WALF iteration is obtained as

$$
b_{i+1}(t)=\left(W_{\varphi i} U_{i}\right)(t)
$$

The WALF method provides path groups with similar time-delay and Doppler-scaling factors. Thus, it will require a much smaller set of time-scale parameters for the WSF estimation, resulting in a computationally much less expensive procedure. The dictionary data matrix will be much smaller than the dictionary built without prior knowledge in the least-squares estimation approach. Specifically, once the path groups are identified and extracted using the WALF algorithm, we estimate the WSF coefficients within each path group using the least-squares approach described in Section 2.2. If $x_{i}(t)$ denotes the signal extracted from the $i$ th path group, $i=1, \ldots, L$, then we can rewrite the received signal as

$$
x(t)=\sum_{i=1}^{L} x_{i}(t)
$$

Applying the discrete time-scale representation of the channel in (2) within each path group leads to

$$
x_{i}(t)=\sum_{m=M_{0}}^{M_{1}} \sum_{n=0}^{N_{i}(m)} \Psi_{n, m}^{(i)} \eta_{0}^{m / 2} s\left(\eta_{0}^{m} t-\frac{n}{W}\right)
$$

where $\Psi_{n, m}^{(i)}$ are the $i$ th path group WSF coefficients and $N_{i}(m)$ depends on the time-delay spread $T_{\text {delay }}(i)$ of the $i$ th path group. One advantage of this approach is that each path group corresponds to a time-delay spread that is much smaller than the overall channel time-delay spread

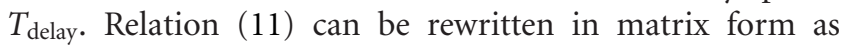
$\mathbf{x}_{i}=\mathbf{D}_{i} \Psi^{(i)}$, where $\mathbf{D}_{i}$ is a signal matrix whose columns consist of time-shifted and scale-changed versions of the transmitted signal $s(t)$, and $\Psi^{(i)}$ is a row vector whose values are the WSF coefficients of the $i$ th path group. In order to minimize the received signal reconstruction error, we propose to estimate the WSF coefficients using the leastsquares estimation method

$$
\hat{\boldsymbol{\Psi}}^{(i)}=\left(\mathbf{D}_{i}^{T} \mathbf{D}_{i}\right)^{-1} \mathbf{D}_{i}^{T} \mathbf{x}_{i}
$$

where $\hat{\boldsymbol{\Psi}}^{(i)}$ is the estimate of $\boldsymbol{\Psi}^{(i)}$. Using (2), (10), and (11), the overall WSF estimate of the received signal is given by

$$
\hat{\Psi}_{n, m}=\sum_{i=1}^{L} \hat{\Psi}_{n, m}^{(i)} .
$$

Note that the signal dictionaries used for estimating the WSF coefficients of the path groups are actually part of the larger dictionary that represents all the transformations undergone by the received signal whose elements are expressed in (2). Thus, only one signal dictionary has to be computed to complete the received signal WSF coefficients estimation.

\section{Ray Theory Model Channel Characterization}

3.1. Ray Theory Model. When the communication channel is sparse, we expect a lot of the discrete WSF values $\Psi_{n, m}$ in (2) to be zero. As a result, it would be computationally intensive to try and estimate the WSF, even for multiple ray groups. Following the ray theory model, the received signal is characterized by a summation of propagating rays, where each ray arrives with a distinctive time delay and a distinctive Doppler scale due to the channel's physical propagation properties $[17,28]$. Specifically, using ray theory, the (noiseless) received signal can be represented as [17]

$$
x(t)=\sum_{i=1}^{N} a_{i} \sqrt{\eta_{i}} s\left(\eta_{i}\left(t-\tau_{i}\right)\right),
$$

where $N$ is the number of propagating ray paths, and $a_{i}, \tau_{i}$, and $\eta_{i}$ are the attenuation factor, time-delay, and Doppler-scale change parameters associated with the $i$ th ray, respectively. When the source is moving at a constant speed $V$, the Doppler scale of the $i$ th ray satisfies

$$
\eta_{i}=\frac{1}{\left[1-(V / c) \cos \left(\theta_{i}\right)\right]},
$$

where $\theta_{i}$ is the declination angle of the $i$ th ray and $c$ is the speed of sound in the medium [17]. Note that the ray theory based signal representation in (14) can be shown to be a special case of the time-scale system characterization in (1), with a highly localized WSF in the time-scale plane that is given by

$$
\mathcal{X}(\tau, \eta)=\mathcal{X}_{\text {ray }}(\tau, \eta)=\sum_{i=1}^{N} a_{i} \delta\left(\tau-\tau_{i}\right) \delta\left(\eta-\eta_{i}\right),
$$


where $\delta(\cdot)$ is the Dirac delta function. For realistic underwater acoustic channels, the WSF can be approximated to have the form in (16) for high frequency cases. In general, however, the time-scale representation in (1) and its discrete version in (2) provide more accurate models for received signals.

3.2. MPD-Based WSF Estimation. Due to the highly localized WSF assumption in (16), the MPD can be used to determine the time-scale features associated with the channel. The MPD is an iterative algorithm that expands a signal into a weighted linear combination of elementary functions (or atoms) chosen from a complete dictionary. It was originally proposed to decompose any finite energy signal as a linear expansion of time-shifted, frequency-shifted, and scaled Gaussian functions [35]. The MPD was later modified to adapt to the analysis signal by changing the atoms to match the analysis signals or by changing the time-frequency signal transformations, as long as the transformations are complete [43]. For this application, the atoms need to match the wideband signal basis functions so that the MPD can provide information on the channel reduced attribute parameters in terms of time shifts, scale changes, and attenuation factors.

The MPD is an iterative algorithm that expands the received finite energy signal $x(t)$ as

$$
x(t)=\sum_{i=0}^{L-1} \alpha_{i} g_{i}(t)+r_{L}(t),
$$

where $g_{i}(t)$ is the basis function selected at the $i$ th iteration and $\alpha_{i}$ is the corresponding expansion coefficient. After $L$ MPD iterations, the residue signal $r_{L}(t)$ is such that the original signal energy is preserved, that is $\|x\|_{2}^{2}=$ $\sum_{i=0}^{L-1}\left|\alpha_{i}\right|^{2}+\left\|r_{L}\right\|_{2}^{2}$ where $\|x\|_{2}^{2}=\int|x(t)|^{2} d t$. In order to best fit the underwater acoustic model with matched timescale transformations, the dictionary atoms $g^{(m, n)}(t) \in \mathscr{D}$ are designed to match time-delayed and Doppler-scaled versions of the transmitted signal $s(t)$ as in (4).

At the beginning of the iterative process, $r_{0}(t)=x(t)$. At the $i$ th iteration, $i=0,1, \ldots, L-1$, the projections of the residue $r_{i}(t)$ onto every dictionary element $g^{(m, n)}(t)$ are computed. The selected dictionary atom $g_{i}(t)$, with parameters $\eta_{i}$ and $\tau_{i}$, is the one that maximizes the magnitude of the projection; its corresponding expansion coefficient is given by $\alpha_{i}=\left\langle r_{i}, g_{i}^{(m, n)}\right\rangle=\int_{-\infty}^{+\infty} r_{i}(t) g_{i}^{(m, n) *}(t) d t$. Note that the residues at the $i$ th and $(i+1)$ th iterations are related as $r_{i+1}(t)=r_{i}(t)-\alpha_{i} g_{i}(t)$. The extracted sparse underwater acoustic signal characteristics are then the MPD parameters $\left(\alpha_{i}, \eta_{i}, \tau_{i}\right), i=0, \ldots, L-1$.

In order to obtain a compact MPD representation for characterizing underwater acoustic channels, it is important to compute the dictionary $\mathscr{D}$ for the appropriate range of time delays and scale changes. Thus, we consider the Doppler tolerance (i.e., half-power contour) of known signals propagating in the channel and then decide on the range of the scale change parameter according to this tolerance. For example, if a linear frequency-modulated (LFM) signal is transmitted, the Doppler tolerance is $[17,44,45]$

$$
V_{D}= \pm 2610 \frac{1}{T W} \text { knots, }
$$

where $T$ is the duration and $W$ is the bandwidth of the LFM signal. As the scale change parameter is affected by the source velocity, the velocity sampling rate is chosen as

$$
\delta v=\frac{V_{D}}{2} \text {. }
$$

We assume that the expected velocities are bounded by $v \in$ $\left[V_{\min }, V_{\max }\right]$ and the expected time delays are bounded by $\tau \in\left[0, T_{\text {delay }}\right]$, where $T_{\text {delay }}$ is the time delay spread of the channel. Then, the signals in the dictionary $\mathscr{D}$ are obtained to match the transmitted signal $s(t)$ according to

$$
g_{m, n}(t)=\left(1-\frac{v_{m}}{c}\right)^{-1 / 2} s\left(\frac{1}{1-v_{m} / c}\left(t-\tau_{n}\right)\right),
$$

where $v_{m}=V_{\min }+0.5 m V_{D}, \tau_{n}=n / f_{s}, f_{s}$ is the sampling frequency, and the integers $m$ and $n$ satisfy $m \in\left[1,2\left(V_{\max }-\right.\right.$ $\left.\left.V_{\min }\right) / V_{D}\right]$ and $n \in\left[1, f_{s} T_{\text {delay }}\right]$.

As the MPD is recursive, the residual energy can be used to determine the algorithm's stopping criteria. If the signal-to-noise ratio (SNR) is known, then the MPD can stop iterating when the ratio of the signal energy to the residual energy reaches the SNR. Other plausible stopping criteria include the rate of decrease of the residual energy or a fixed number of iterations based on prior knowledge of the range of values of the channel parameters. When a known OFDM signal is transmitted, the same implementation is applied for signal characterization, and we empirically use the velocity parameter sampling rate obtained from an LFM signal, which has the same time duration and frequency bandwidth as the OFDM signal. As this velocity parameter sampling rate is much finer than the sampling rate obtained from the discrete time-scale representation approach, we conclude that this dictionary $\mathscr{D}$ is complete for decomposing the received signal.

\section{Underwater Acoustic Channel Estimation for the KAM08 Experiment}

4.1. KAM08 Experiment Description. The experimental data were collected during the KAM08 experiment [46], which was conducted in shallow water off the western coast of Kauai, Hawaii, in June 2008. We present results for a towedsource scenario immersed at depth spanning $20-50 \mathrm{~m}$ and towed at a constant speed of 3 knots (about $1.5 \mathrm{~m} / \mathrm{s}$ ). The receiver was a fixed 16-element vertical array, as illustrated in Figure 1, with a $50 \mathrm{kHz}$ sampling rate. The interelement spacing was $3.75 \mathrm{~m}$, with the top element deployed at a nominal depth of $42.5 \mathrm{~m}$. We focus on the results obtained by processing the data recorded at the 5 th receiving element, whose depth was $83.5 \mathrm{~m}$, for a source moving toward the fixed receiver at about $24 \mathrm{~m}$ depth. The source receiver separation was approximately $1.5 \mathrm{~km}$. The bathymetry of the operation area is shown in Figure 2 [46]. 


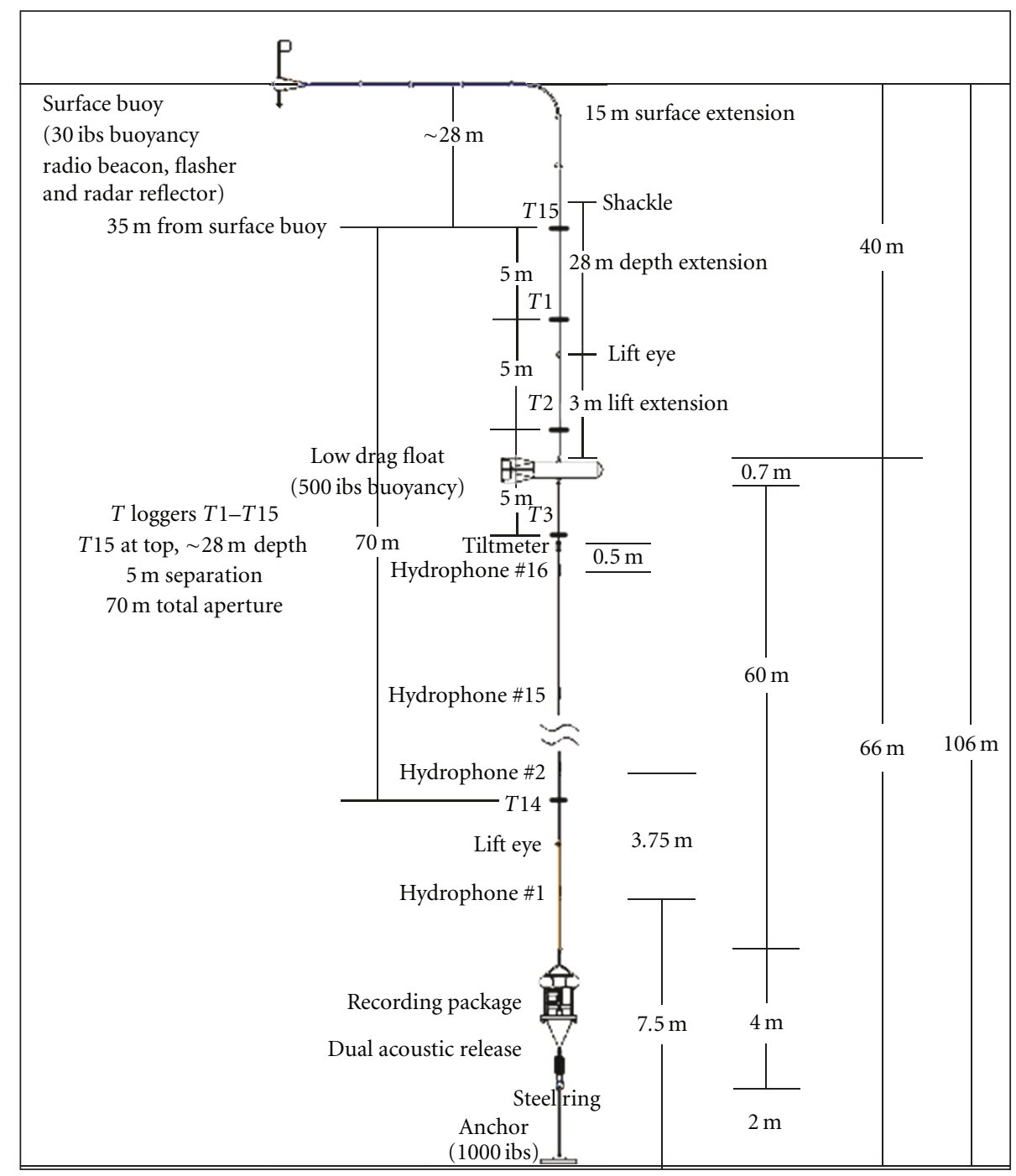

FIGURE 1: Scheme of the vertical array receiver adapted in the KAM08 experiment [46].

The transmitted waveforms are OFDM signals modulated with binary phase shift keying (BPSK) symbols and LFM probing signals. Both LFM and OFDM signals have a $16 \mathrm{kHz}$ central frequency and an $8 \mathrm{kHz}$ bandwidth. These are wideband signals as their central frequencies are comparable to their bandwidth. The LFM signal has $100 \mathrm{~ms}$ time duration and starts at the end of the communication blocks. The OFDM signals incorporate 1024 subcarriers, with a $7.8 \mathrm{~Hz}$ subcarrier spacing, a $256 \mathrm{~ms}$ frame length, and a $20 \mathrm{~ms}$ cyclic prefix. The communication blocks consist of 8 frames, followed by one LFM probing signal. The spectrogram of a received communication frame consisting of 8 communication blocks and followed by an LFM signal is demonstrated in Figure 3.

\subsection{WSF Estimation Using the Discrete Time-Scale Represen-} tation Approach. As we discussed in Section 2, the WSF of an underwater acoustic channel can be estimated using the discrete time-scale representation either directly, using the least-squares approach or using the WALF method if the WSF forms path groups. Figure 4 shows the WAF of the received LFM probing signal. Here, the speed parameter is plotted instead of the scale parameter as the two parameters are related (see, e.g., their relationship in (20)). Applying the WALF algorithm to the received LFM probing signal, we were able to identify and estimate three path groups. The three detected path groups are marked by crosses representing the delay speed coordinates in the WAF domain in Figure 4. The estimated WSF coefficients of the three path groups are shown in Figure 5. The WALF algorithm estimated and extracted three arrivals path groups in the received signal, representing $73.2 \%$ of the total signal energy. One WSF was estimated for each extracted arrival path group with delay and scaling parameters centered around the WALF estimates.

The Mellin-domain support parameter $\beta_{0}$ of the transmitted LFM signal was needed in order to compute the WSF coefficients for each path group. This parameter was computed as $\beta_{0}=\left(f_{0}+W / 2\right) T$ [47], where $f_{0}, W$, and $T$ are 


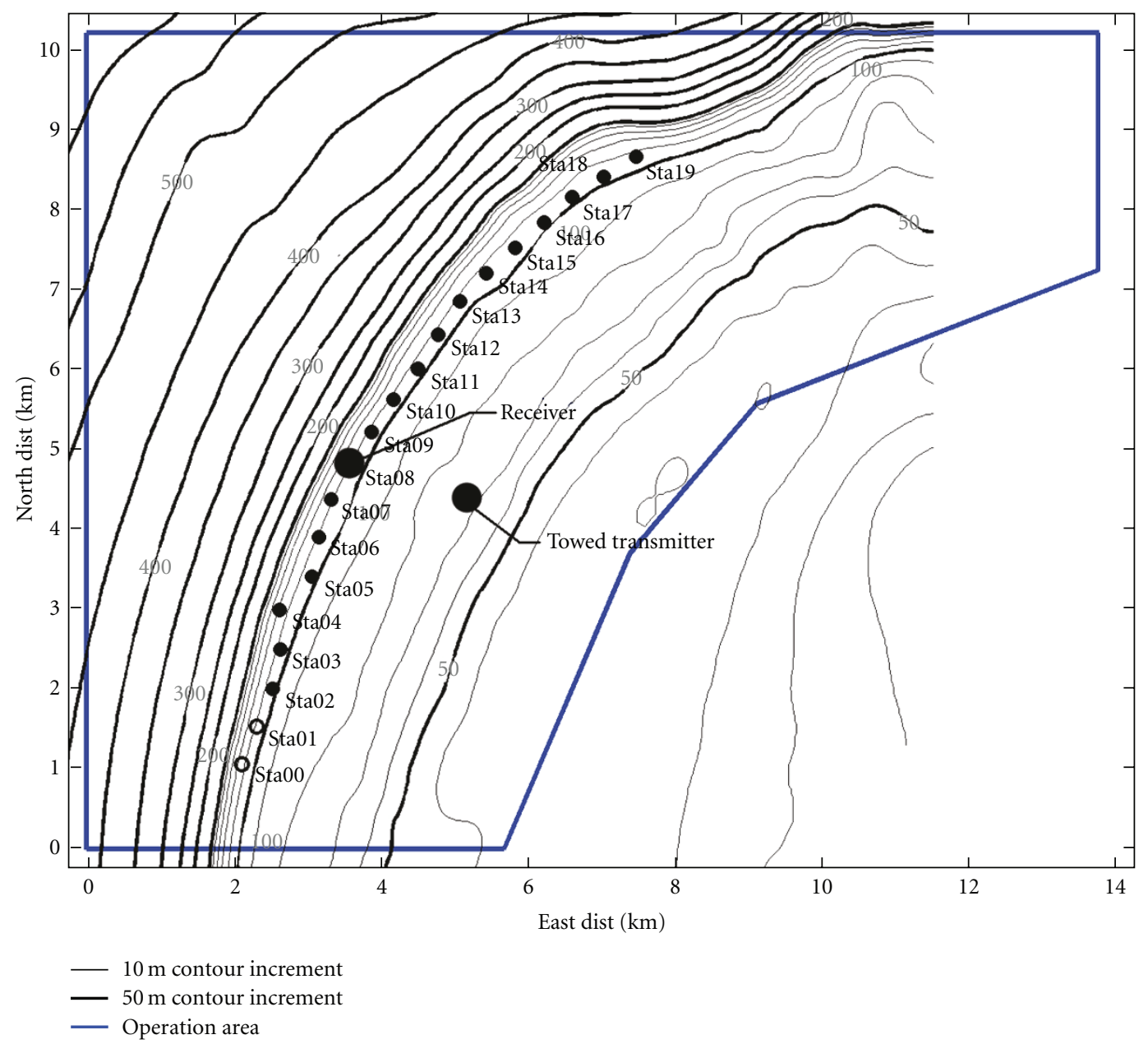

FIGURE 2: Bathymetry of the operation area (with the depth given in meters), receiver and transmitter positions used in the KAM08 experiment [46].

the LFM signal central frequency, bandwidth, and duration of the LFM signal. Using (2), we were able to characterize the discrete WSF of the channel with a minimum number of time-shifts and Doppler-scaling parameters. The timedelay spread within each path group was considered to be $10 \mathrm{~ms}$, resulting in 81 time shifts. For this experimental data set, since $\eta_{0}=1.008$ was close to 1 , the number of time shifts was the same for all different scale factors. The Doppler spread considered within each path group was computed using (19) to be $\delta v= \pm 1.6 \mathrm{~m} / \mathrm{s}$, resulting in 2 Doppler scale factors. The WSF recovery errors within each path group were, respectively, $\varepsilon_{1}=0.91 \%$, $\varepsilon_{2}=0.42 \%$, and $\varepsilon_{3}=0.76 \%$. The overall recovery error of the signal $x_{e}(t)$ extracted using the WALF approach was $\epsilon_{e}=$ $\int\left|x_{e}(t)-\hat{x}(t)\right|^{2} d t / \int\left|x_{e}(t)\right|^{2} d t=0.87 \%$, where $\hat{x}(t)$ is the recovered signal using the WSF estimate in (13). Combining the WALF algorithm extraction percentage and the discrete WSF recovery error, the overall recovery error on the received signal $x(t)$ is $\epsilon=\int|x(t)-\hat{x}(t)|^{2} d t / \int|x(t)|^{2} d t=23.8 \%$, where $\hat{x}(t)$ is the recovered signal using the WSF estimate in (13).
Figures 4 and 5 show that the different path groups have different time delays and Doppler scales, as indicated by both the WAF and the discrete WSF. The WSF representation in Figure 5 also shows that the first direct arrival path group is well localized in the delay-domain, whereas the next arrival paths are bouncing off the sea bottom vor sea surface and are more scattered along the delay domain. This demonstrates that the discrete WSF is well adapted to the physical nature of the channel distortions.

Using the discrete WSF and the least-squares estimation approach with the received OFDM signal, we estimated the channel WSF coefficients as shown in Figure 6. Note that we could not use the WALF approach with OFDM signals due to the discontinuities in their phase functions. For OFDM signals, the theoretical Mellin-domain support is not known. However, we experimentally obtained it by computing the Mellin transform of the OFDM signal to be almost twice the value of the Mellin-domain support of the LFM signal. Hence, we considered twice as many Doppler scale factors (i.e., six scale factors) for the OFDM signal as we did for the LFM signal for the same Doppler spread. From the 


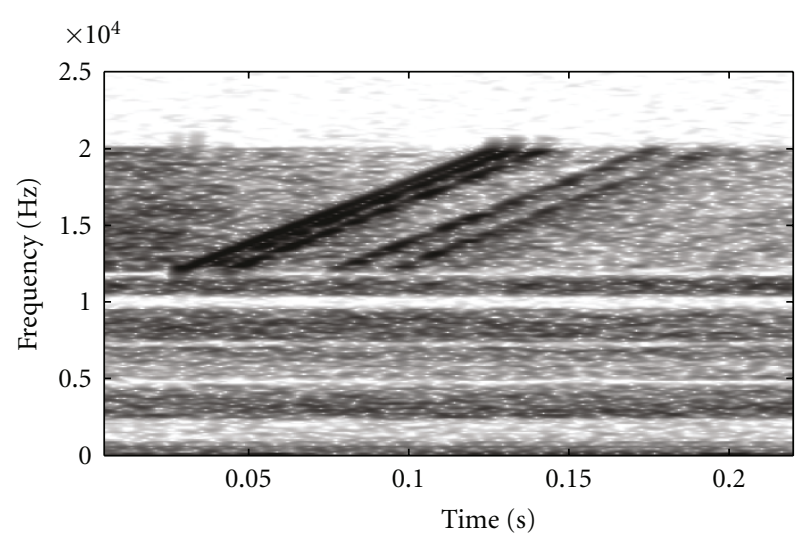

Figure 3: Spectrogram time-frequency representation of the received communication and probing LFM signals transmitted by the towed source in the KAM08 experiment, demonstrating the effects of the multipath underwater propagation (especially the time-delayed arrivals of the LFM signal).

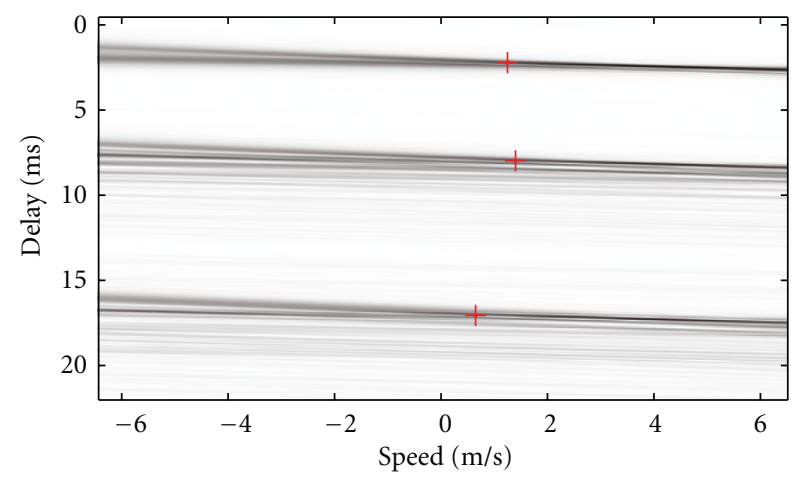

FIgURE 4: Wideband ambiguity function of the received LFM probing signal in the KAM08 experimental data. The crosses represent the delay-speed coordinates of the three detected path groups.

results obtained with the LFM signal, the channel spread was estimated to be $20 \mathrm{~ms}$, resulting in 269 time shifts. The channel WSF estimate obtained using the OFDM signal in Figure 6 is consistent with that obtained using the LFM signal. We clearly estimated three main path groups, with the same time-delay spread characteristics and comparable Doppler scaling estimates. The recovery error for the whole signal was $\varepsilon=24.2 \%$ which is consistent with the results obtained with the LFM signal.

4.3. WSF Estimation Using the MPD. As the channel characteristics are sparse, we also applied the MPD algorithm to the LFM probing signals to estimate the channel. The towed source speed is considered unknown and is estimated during the first MPD iteration to be $V=1.2 \mathrm{~m} / \mathrm{s}$ using (15). For this application, the signal Doppler tolerance is computed using (18) to be $V_{D}= \pm 1.6 \mathrm{~m} / \mathrm{s}$. The speed range is obtained from $V_{\min }=V-V_{D}=-0.4 \mathrm{~m} / \mathrm{s}$ to $V_{\text {max }}=V+V_{D}=2.8 \mathrm{~m} / \mathrm{s}$ and the number of scaling factors is computed using (19), leading to $\delta v=0.8 \mathrm{~m} / \mathrm{s}$ and 5

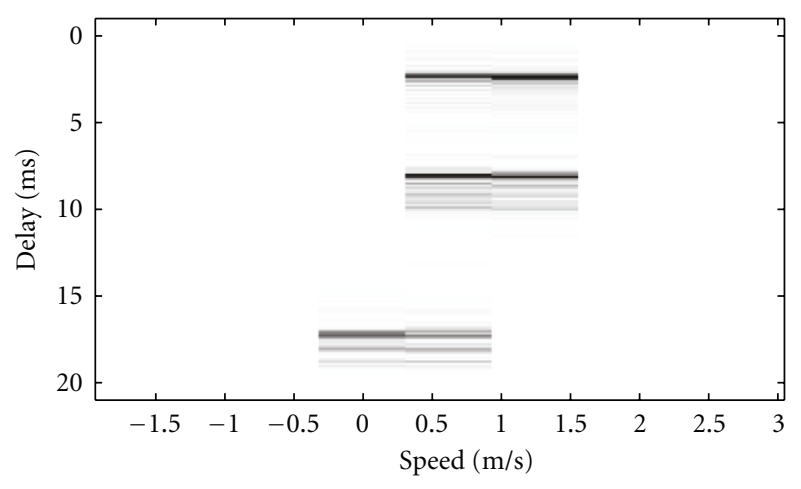

FIGURE 5: WSF estimated coefficients of the three detected path groups using the WALF method and the received LFM probing signal in the KAM08 experimental data.

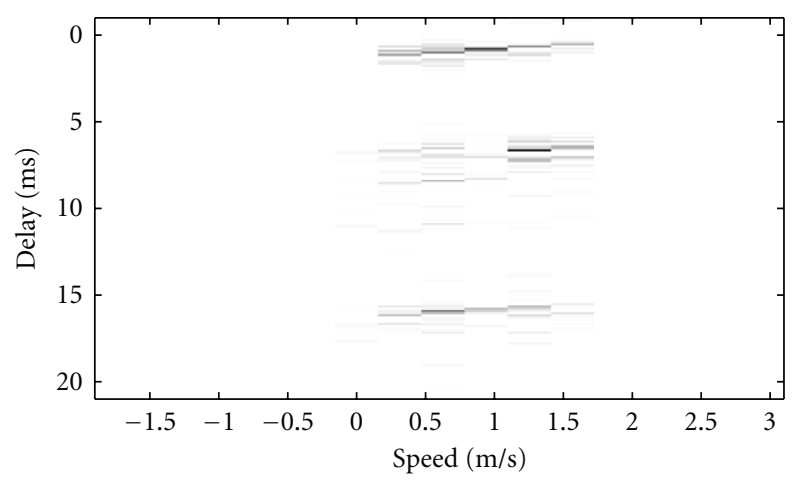

FIGURE 6: WSF estimated coefficients using the least-squares method and the received OFDM probing signal in the KAM08 experimental data.

different Doppler scaling factors. Figure 7 shows the WAF of the received LFM signal together with the sparse and highly localized channel time-scale features extracted after $L=$ 50 MPD iterations. The estimated delay and Doppler scale parameters are represented with crosses, thus demonstrating the sparsity of the channel. The recovery error obtained after $50 \mathrm{MPD}$ iterations is only $\varepsilon=13.4 \%$, proving that the sparse time-scale model is well adapted to characterize the underwater acoustic propagation. This result is better than the one obtained using the WSF method (where the recovery error was $\varepsilon=23.8 \%$ ). For the WSF results, we only considered the first 3 strongest path groups. However, for the MPD approach, we did not have this constraint; we ended up using 5 ray groups and thus achieving higher performance. The MPD underwater acoustic channel estimate is also very sparse as only 50 dictionary signals were necessary to characterize the channel properly, while 486 dictionary signals were used with the WSF characterization. Figure 8 represents a zoomed-in version around the first 3 arrival groups of Figure 7, showing that the first and direct arrival group is well localized in delay, whereas the next arrival groups are bouncing off the sea bottom or sea surface and are more scattered. 


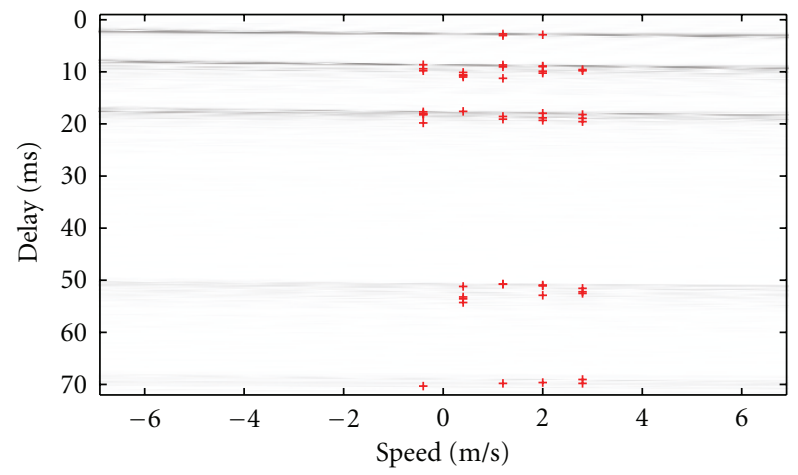

FIGURE 7: Wideband ambiguity function of the received LFM signal in the KAM08 experimental data. The crosses represent the maxima detected after each MPD iteration.

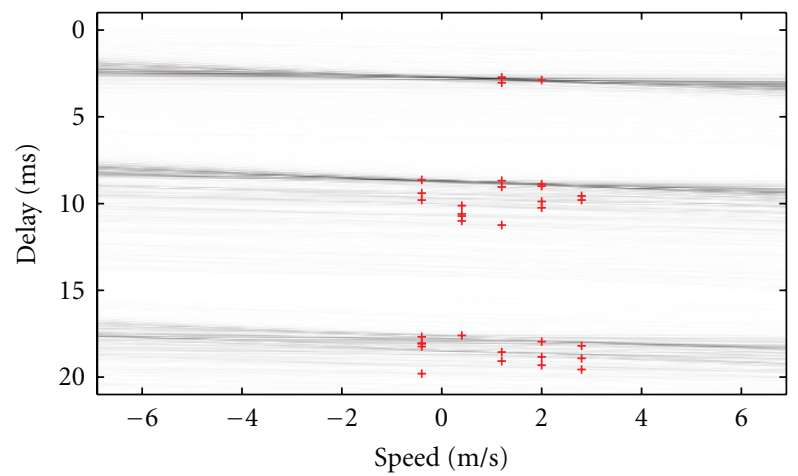

FIGURE 8: Zoomed-in version of Figure 7 around the first three arrival arrival groups in the wideband ambiguity function of the received LFM signal in the KAM08 experimental data. The crosses represent the maxima detected after each MPD iteration.

Using the sparse time-scale channel representation for the received OFDM signal, we estimated the sparse channel time-scale features as shown in Figure 9. The speed estimate and the range of possible speeds considered for the OFDM signal are the same as the ones chosen for the LFM signal. As the Doppler tolerance of the OFDM signals was not known, we used the WSF Mellin-based approach [20] to determine the number of Doppler scaling factors; this resulted in $\delta v$ $0.3 \mathrm{~m} / \mathrm{s}$ and 12 Doppler scales. Figure 9 shows the WAF of the received OFDM signal together with the sparse channel time-scale features extracted after $L=130$ MPD iterations. The recovery error was $\varepsilon=24 \%$ after 50 MPD iterations and $\varepsilon=14.7 \%$ after $130 \mathrm{MPD}$ iterations. The results obtained with OFDM signals are consistent with the LFM results even though the OFDM signals required more MPD iterations to obtain the same recovery error. Note that only 130 dictionary signals were needed to characterize the channel properly; when the WSF discrete time-scale model was used, 1345 dictionary signals were used to estimate the WSF coefficients. Figure 10 shows a zoomed-in version of Figure 9 around the third arrival group, illustrating the channel time-scale feature spread of one arrival group.

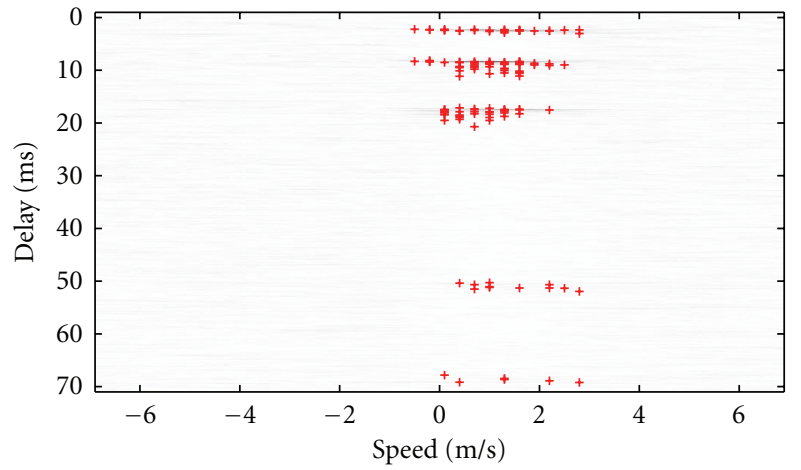

Figure 9: Wideband ambiguity function of the received OFDM signal in the KAM08 experimental data. The crosses represent the maxima detected after each MPD iteration.

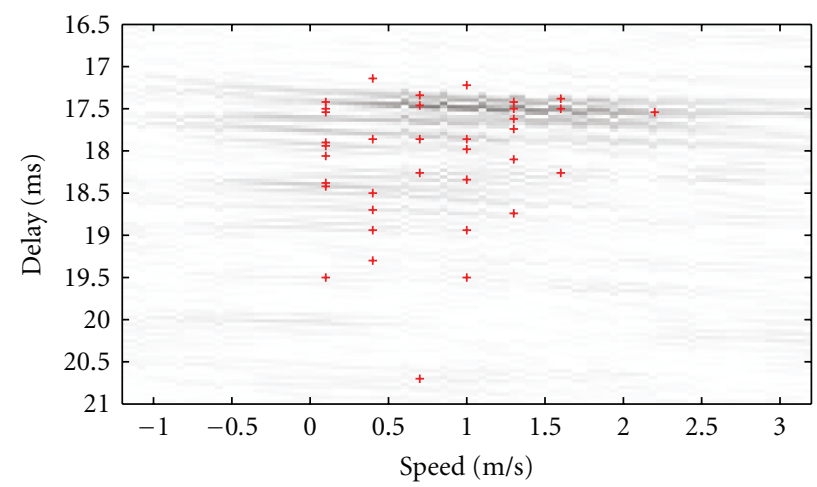

FIgURE 10: Zoomed-in version of Figure 9 around the third arrival path group in the wideband ambiguity function of the received OFDM signal in the KAM08 experimental data. The crosses represent the maxima detected after each MPD iteration.

4.4. Multipath Profile Extracted from Signal Characteristics. Another central challenge in shallow underwater acoustic communications is the temporal evolution of the underwater acoustic channel. Recently, in [34], underwater acoustic channel rapid fluctuations were estimated over time using the time-frequency spreading function and the MPD, following the narrowband approximation. Here, we no longer assume narrowband conditions and we use the wideband MPD-based channel characterization in order to illustrate the source motion effects on the channel multipath profile.

Figure 11 presents the three-dimensional (3D) channel profile evolution over time, and Figure 12 shows its 2D projection on the time-delay domain for clarity. It was computed using the MPD-based channel characterization for communication and LFM signals from the KAM08 experiment. We considered a signal consisting of 8 frames, each followed by one LFM probing signal and a $0.1 \mathrm{~s}$ of silence. For each transmission frame, the signal characteristic parameters were provided by 50 MPD iterations for the LFM signals, and by $130 \mathrm{MPD}$ iterations for the OFDM signals. As we can see, the multipath profile evolves over time as the range between the transmitter and the receiver changes. 


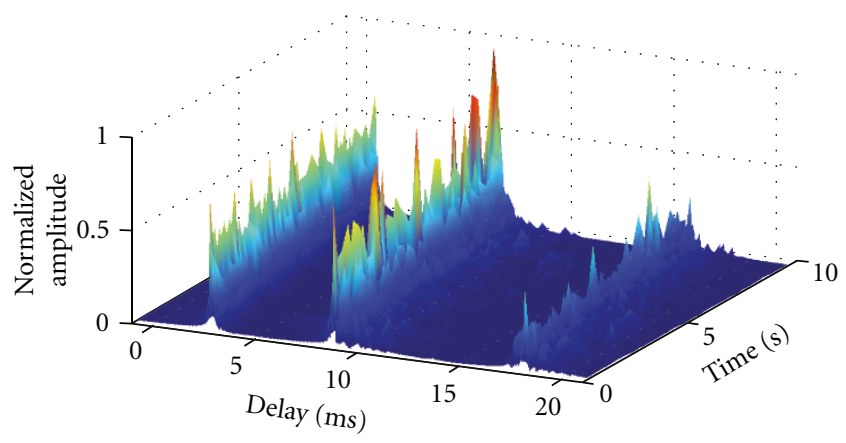

Figure 11: Three-dimensional representation of the underwater acoustic channel estimation over time, zoomed-in around the first three arrival path groups for the KAM08 experiment.

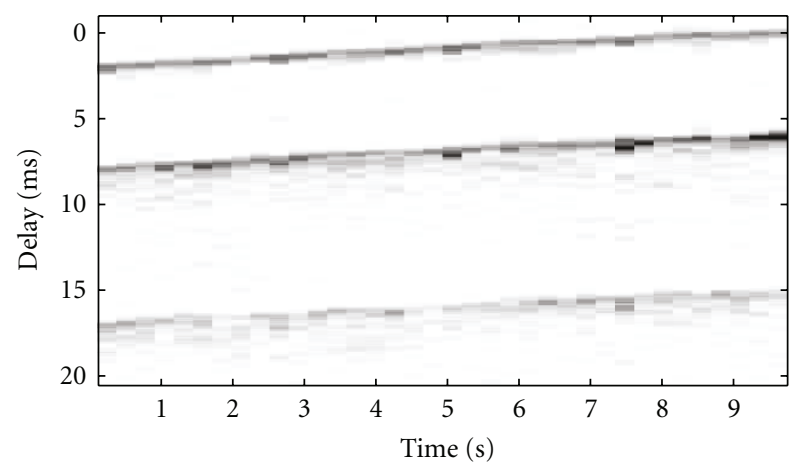

Figure 12: Two-dimensional representation of the underwater acoustic channel estimation over time, zoomed-in around the first three arrival path groups for the KAM08 experiment.

Hence, the time delays associated with the different arrival path groups evolve over time, as illustrated in Figure 12. In our example, the source is approaching at a constant speed so the source receiver separation decreases linearly, explaining the linear time-delay variations in Figure 12. Figure 11 also exhibits attenuation coefficient variations over time due to the time-varying changes of the propagation environment as the source moves.

\section{Underwater Acoustic Channel Estimation for the BASE07 Experiment}

5.1. BASE07 Experiment Description. The BASE07 experiment was jointly conducted by the NATO Undersea Research Center (NURC), the Forschungsanstalt der Bundeswehr für Wasserschall und Geophysik (FWG), the Applied Research Laboratory (ARL), and the Service Hydrographique et Ocanographique de la Marine (SHOM). The main objective of the experiment was to investigate broadband adaptive sonar techniques in shallow water [17,27,37]. The campaign took place on the Malta Plateau in shallow water $(130 \mathrm{~m}$ depth). Underwater acoustic LFM signals, as illustrated in Figure 13, were transmitted by a source moving rectilinearly at a constant speed, from 2 to 12 knots and at different depths. The transmitted LFM signals had a $2 \mathrm{kHz}$ bandwidth,

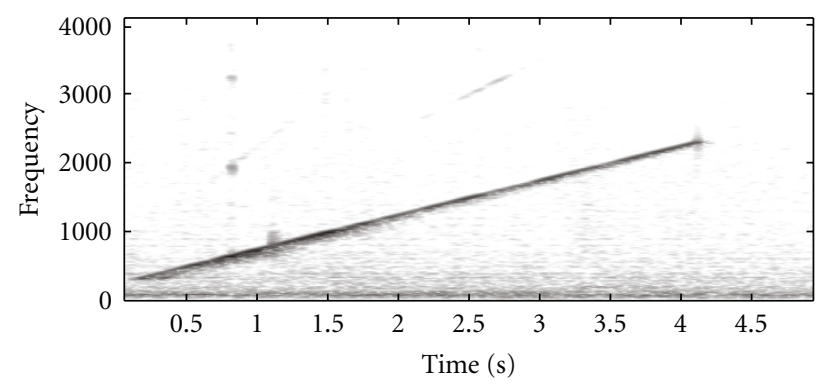

Figure 13: Spectrogram of the LFM signal transmitted by the towed source, demonstrating the multipath effects of underwater propagation for the BASE07 experiment.

$1.3 \mathrm{kHz}$ central frequency, and $4 \mathrm{~s}$ duration; the effects of the multipath propagation can be seen in Figure 13. The bandwidths of the transmitted signals were very large compared to the central frequency. Thus, the signals were Doppler sensitive and had a very low Doppler tolerance. The transmitter-receiver range varied from 400 to $25,000 \mathrm{~m}$ and the transmitted signals were recorded by an array of six hydrophones located at different depths (from 9 to $94 \mathrm{~m}$ ). As shown in Figure 14, the array of hydrophones had its own global positioning system (GPS) and clock for localization and was not anchored so it could move freely with currents and avoid additional flow noise. The boat had a GPS which was used to obtain the position of the towed transmitter. Both position and speed of the source and the hydrophone array were known at any moment so the results could be compared and analyzed. In the following, the presented scenario was recorded on the hydrophone located at $42 \mathrm{~m}$ depth, with a $490 \mathrm{~m}$ source receiver separation. Also, both the source and the receiver were moving with a relative velocity of about $0.95 \mathrm{~m} / \mathrm{s}$.

5.2. WSF Estimation Using the Discrete Time-Scale Representation Approach. The WAF of the received LFM signal is illustrated in Figure 15. The WALF algorithm was applied to the received LFM signal to estimate the WSF coefficients, and the crosses in Figure 15 indicate the parameters of the four estimated path groups. The towed source speed is considered unknown and is estimated during the first WALF iteration to be $V=0.9 \mathrm{~m} / \mathrm{s}$ using (15). Figure 16 shows the resulting estimated WSF coefficients from the four path groups. The WALF algorithm estimated and extracted 4 arrival path groups in the received signal with $80.76 \%$ of the total signal energy. The time-delay spread considered within each path group was $30 \mathrm{~ms}$, leading to 61 time shifts. For this experimental data set, the number of time shifts was also the same for all different scale factors since $\eta_{0}=1.0001$ is very close to 1 . Hence, the dictionary necessary to characterize the received LFM signal consisted of 488 time-delayed and scaled versions of the transmitted signal.

The Doppler-scale spread considered within each path group was computed using (19) to be $\delta v= \pm 0.16 \mathrm{~m} / \mathrm{s}$, resulting in 2 Doppler scale factors. The WSF recovery error within each path group was $\varepsilon_{1}=3.59 \%, \varepsilon_{2}=6.04 \%$, 

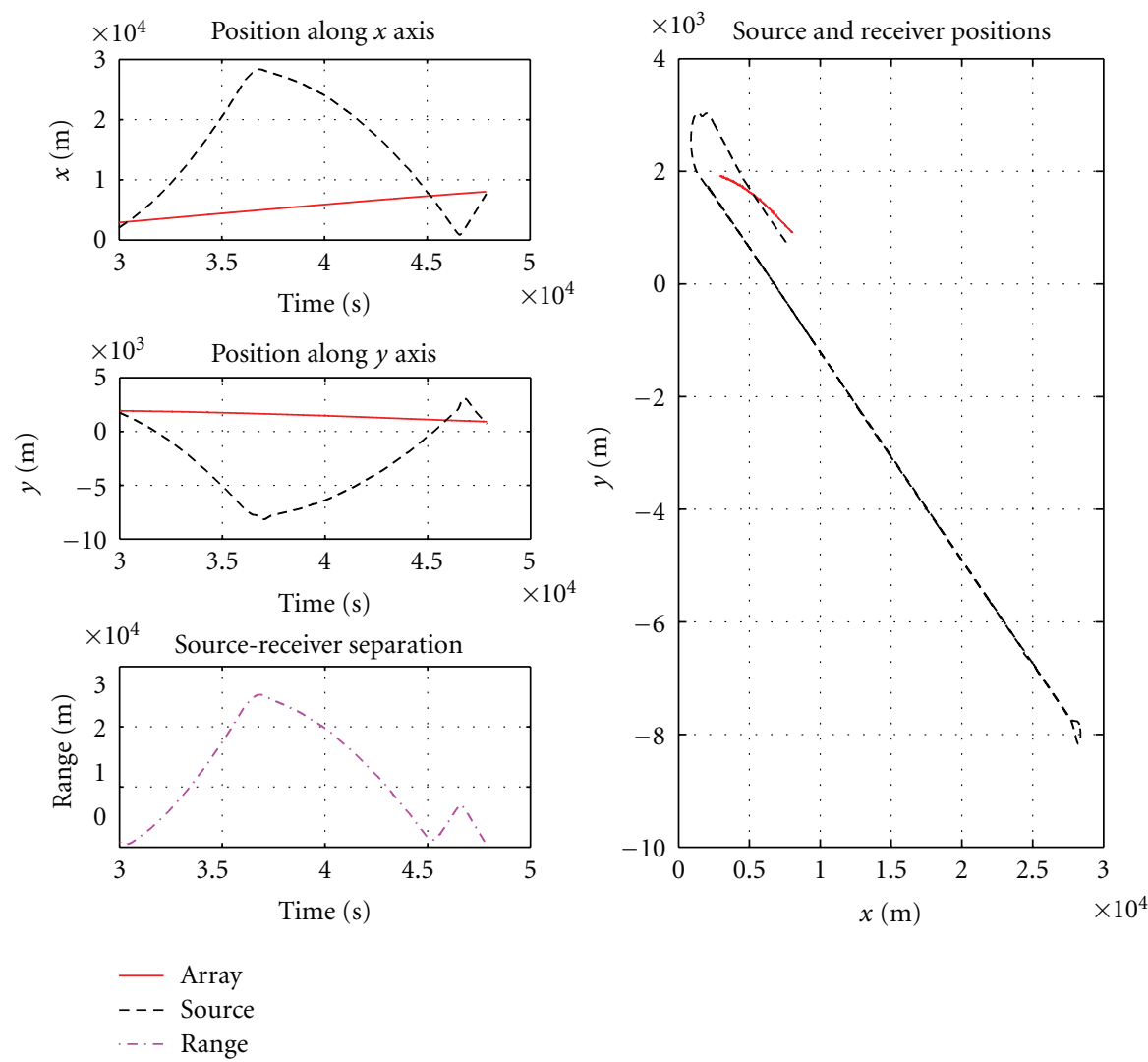

FIGURE 14: Positions of the towed source and of the hydrophones along time and evolution of the source-receiver separation along time for the BASE07 experiment.

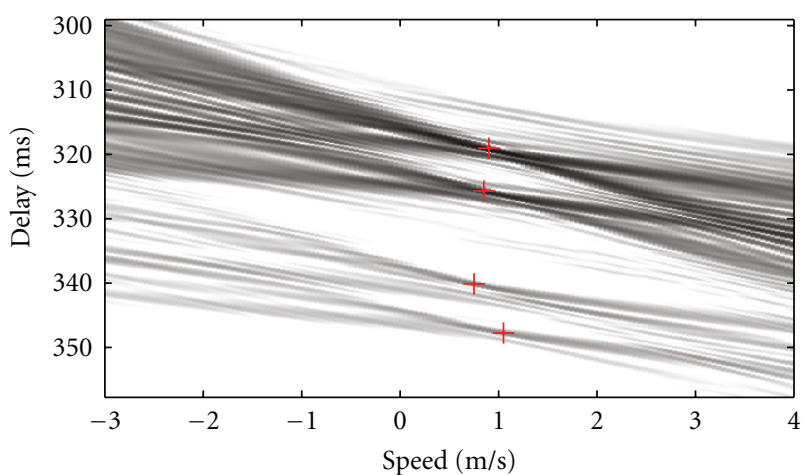

FIGURE 15: Wideband ambiguity function of the received LFM signal in the BASE07 experimental data. The crosses represent the positions of the four detected path groups.

$\varepsilon_{3}=26.12 \%$, and $\varepsilon_{4}=18.31 \%$; the total recovery error was $\varepsilon=$ $5.27 \%$ on the extracted signals. Note that most of the received signal energy was contained within the first two arrival paths; the last two arrival paths had a lower SNR and thus were more difficult to characterize. The total recovery error was found to be $\varepsilon=24.51 \%$. Figures 15 and 16 show that the different path groups had different time-delay and Dopplerscale factors; this was taken into account in the discrete WSF signal characterization.

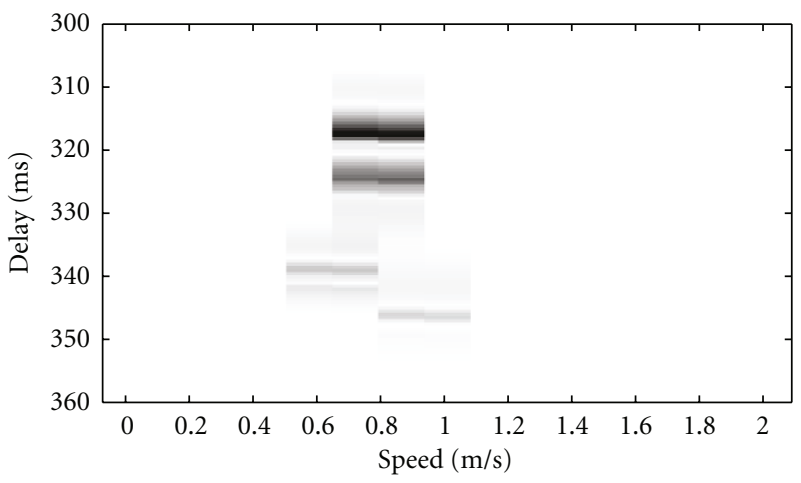

FIGURE 16: WSF estimated coefficients of the four detected path groups using the WALF method and the received LFM signal in the BASE07 experimental data.

5.3. WSF Estimation Using the MPD. The MPD-based algorithm was also applied to characterize the underwater acoustic signals from the BASE07 experiment, as shown in Figure 17. The towed source speed was considered unknown and was estimated during the first MPD iteration to be $V=$ $0.9 \mathrm{~m} / \mathrm{s}$ using (15). For this application, the signal Doppler tolerance was low and was computed using $(18)$ to be $V_{D}=$ $\pm 0.16 \mathrm{~m} / \mathrm{s}$. The speed range was chosen from $V_{\min }=V-2 V_{D}$ $=0.58 \mathrm{~m} / \mathrm{s}$ to $V_{\max }=V+2 V_{D}=1.22 \mathrm{~m} / \mathrm{s}$. The number 


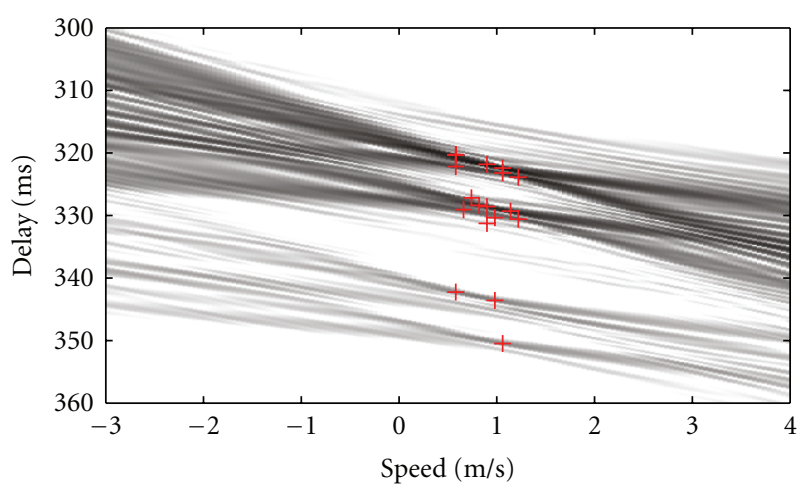

FIgure 17: Wideband ambiguity function of the received LFM signal in the BASE07 experimental data. The crosses represent the maxima detected after the first 20 MPD iterations.

of scaling factors was computed to be $\delta v=0.08 \mathrm{~m} / \mathrm{s}$ with nine different Doppler scaling factors using (19). Note that the LFM signals transmitted during the BASE07 experiment had low Doppler tolerance and required numerous scaling factors to provide good signal characterization. In fact, for short range and high speed scenarios, the Doppler scaling factors were often very different between arrival ray groups; this implies that a large dictionary must be used to obtain accurate WSF estimation results. Figure 17 shows the WAF of the received LFM signal together with the highly localized channel time-scale features extracted after 20 MPD iterations. The estimated time-delay and Dopplerscale parameters are represented by crosses, emphasizing the channel sparsity. The recovery error after 50 MPD iterations was $\varepsilon=13.32 \%$. Thus, the highly localized WSF model is well adapted to this experimental scenario. The underwater acoustic signal characterization is also very sparse as only 50 dictionary signals were needed to characterize the channel properly, whereas 488 dictionary signals were used with the WAF estimation method.

\section{Conclusions}

We investigated two approaches for characterizing wideband channels for underwater acoustic communications. Our approaches demonstrate that underwater acoustic signals in the medium-to-high frequency range are characterized by transformations with multiple time delays and multiple Doppler scales, and the parameters for these transformations can be extracted to accurately describe the received signal. The first method employs a discrete timescale system model that represents the received signal in terms of time-shifted and Doppler-scaled versions of the transmitted signal, weighted by the wideband spreading function (WSF). The second method assumes a highly localized wideband spreading function representation and extracts signal characteristics using a matched MPD algorithm. Both methods were successfully validated using OFDM communication signals and LFM signals from two recent experiments. Our results indicate that when the WSF characterization of a wideband underwater acoustic communication channel can be sparsely represented in the lag-Doppler plane, then a time-scale matched representation can provide an accurate and fast channel estimation procedure.

\section{Acknowledgments}

This work was supported in part by Délégation Générale pour l'Armement under SHOM Research Grant N07CR0001 and in part by the Department of Defense MURI Grant no. AFOSR FA9550-05-1-0443. T. Duman's work and the KAM08 experiment was funded by the MURI Grant no. N00014-07-1-0739. The authors would like to thank J. I. Mars (GIT), C. Ioana (GIT), C. Gervaise (ENSIETA), and Y. Stéphan (SHOM) for fruitful discussions.

\section{References}

[1] B. G. Iem, A. Papandreou-Suppappola, and G. Faye Boudreaux-Bartels, "Wideband Weyl symbols for dispersive timevarying processing of systems and random signals," IEEE Transactions on Signal Processing, vol. 50, no. 5, pp. 1077-1090, 2002.

[2] Y. Jiang and A. Papandreou-Suppappola, "Discrete timefrequency characterizations of dispersive linear time-varying systems," IEEE Transactions on Signal Processing, vol. 55, no. 5, pp. 2066-2076, 2007.

[3] A. Papandreou-Suppappola, C. Ioana, and J. J. Zhang, “Timescale and dispersive processing for wideband time-varying channels," in Wireless Communications over Rapidly TimeVarying Channels, F. Hlawatsch and G. Matz, Eds., Academic Press, 2011.

[4] T. Kailath, "Sampling models for linear time-variant filters," Tech. Rep. 352, MIT Research Laboratory of Electronics, Cambridge, Mass, USA, 1959.

[5] P. A. Bello, "Characterization of randomly time-variant linear channels," IEEE Transactions on Communication Systems, vol. 11, pp. 360-393, 1963.

[6] L. J. Ziomek, Underwater Acoustics: A Linear System Theory Approach, Academic Press, New York, NY, USA, 1985.

[7] A. M. Sayeed and B. Aazhang, "Joint multipath-doppler diversity in mobile wireless communications," IEEE Transactions on Communications, vol. 47, no. 1, pp. 123-132, 1999.

[8] Y. Jiang and A. Papandreou-Suppappola, "Time-scale canonical model for wideband system characterization," in Proceedings of the IEEE International Conference on Acoustics, Speech and Signal Processing (ICASSP '05), vol. 4, pp. 281-284, Philadelphia, Pa, USA, 2005.

[9] A. F. Molisch, Wireless Communications, John Wiley \& Sons, Chichester, UK, 2005.

[10] J. G. Proakis, Digital Communications, McGraw-Hill, New York, NY, USA, 4th edition, 2001.

[11] G. B. Giannakis and C. Tepedelenlioglu, "Basis expansion models and diversity techniques for blind identification and equalization of time-varying channels," Proceedings of the IEEE, vol. 86, no. 10, pp. 1969-1986, 1998.

[12] S. Bhashyam, A. M. Sayeed, and B. Aazhang, "Time-selective signaling and reception for communication over multipath fading channels," IEEE Transactions on Communications, vol. 48, no. 1, pp. 83-94, 2000. 
[13] M. Stojanovic and J. Preisig, "Underwater acoustic communication channels: propagation models and statistical characterization," IEEE Communications Magazine, vol. 47, no. 1, pp. 84-89, 2009.

[14] J. J. Zhang, A. Papandreou-Suppappola, B. Gottin, and C. Ioana, "Time-frequency characterization and receiver waveform design for shallow water environments," IEEE Transactions on Signal Processing, vol. 57, no. 8, pp. 2973-2985, 2009.

[15] B. Li, S. Zhou, M. Stojanovic, L. L. Freitag, and P. Willett, "Multicarrier communication over underwater acoustic channels with nonuniform Doppler shifts," IEEE Journal of Oceanic Engineering, vol. 33, no. 2, pp. 198-209, 2008.

[16] N. F. Josso, J. J. Zhang, D. Fertonani, A. PapandreouSuppappola, and T. M. Duman, "Time-varying wideband underwater acoustic channel estimation for OFDM communications," in Proceedings of the IEEE International Conference on Acoustics, Speech, and Signal Processing, pp. 5626-5629, Dallas, Tex, USA, March 2010.

[17] N. F. Josso, C. Ioana, J. I. Mars, C. Gervaise, and Y. Stéphan, "On the consideration of motion effects in the computation of impulse response for underwater acoustics inversion," Journal of the Acoustical Society of America, vol. 126, no. 4, pp. 17391751, 2009.

[18] L. H. Sibul, L. G. Weiss, and T. L. Dixon, "Characterization of stochastic propagation and scattering via Gabor and wavelet transforms," Journal of Computational Acoustics, vol. 2, no. 3, pp. 345-369, 1994.

[19] R. G. Shenoy and T. W. Parks, "Wide-band ambiguity functions and affine Wigner distributions," Signal Processing, vol. 41, no. 3, pp. 339-363, 1995.

[20] Y. Jiang and A. Papandreou-Suppappola, "Discrete time-scale characterization of wideband time-varying systems," IEEE Transactions on Signal Processing, vol. 54, no. 4, pp. 1364-1375, 2006.

[21] S. Rickard, Time-frequency and time-scale representations of doubly spread channels, Ph.D. dissertation, Princeton University, 2003.

[22] A. R. Margetts, P. Schniter, and A. Swami, "Joint scale-lag diversity in wideband mobile direct sequence spread spectrum systems," IEEE Transactions on Wireless Communications, vol. 6, no. 12, pp. 4308-4319, 2007.

[23] Z. H. Michalopoulou, "Matched-impulse-response processing for shallow-water localization and geoacoustic inversion," Journal of the Acoustical Society of America, vol. 108, no. 5, pp. 2082-2090, 2000.

[24] M. I. Taroudakis and G. N. Makrakis, Inverse Problems in Underwater Acoustics, Springer, New York, NY, USA, 2001.

[25] H. Zou, Y. Chen, J. Zhu, Q. Dai, G. Wu, and Y. Li, "Steadymotion-based Dopplerlet transform: application to the estimation of range and speed of a moving sound source," IEEE Journal of Oceanic Engineering, vol. 29, no. 3, pp. 887-905, 2004.

[26] J. P. Hermand and W. I. Roderick, "Delay-Doppler resolution performance of large time-bandwidth-product linear FM signals in a multipath ocean environment," Journal of the Acoustical Society of America, vol. 84, pp. 1709-1727, 1988.

[27] N. F. Josso, J. J. Zhang, A. Papandreou-Suppappola et al., "On the characterization of time-scale underwater acoustic signals using matching pursuit decomposition," in Proceedings of the IEEE of OCEANS Conference, p. 6, Biloxi, Miss, USA, 2009.
[28] N. F. Josso, C. Ioana, C. Gervaise, Y. Stephan, and J. I. Mars, "Motion effect modeling in multipath configuration using warping based lag-Doppler filtering," in Proceedings of the IEEE International Conference on Acoustics, Speech, and Signal Processing (ICASSP '09), pp. 2301-2304, April 2009.

[29] M. Stojanovic, "Low complexity OFDM detector for underwater acoustic channels," in Proceedings of the IEEE of OCEANS Conference, p. 6, Boston, Mass, USA, 2006.

[30] K. Tu, D. Fertonani, T. M. Duman, M. Stojanovic, J. G. Proakis, and P. Hursky, "Mitigation of intercarrier interference for OFDM overtime-varying underwater acoustic channels," to appear in IEEE Journal of Oceanic Engineering.

[31] K. Tu, D. Fertonani, T. M. Duman, and P. Hursky, "Mitigation of intercarrier interference in OFDM systems over underwater acoustic channels," in Proceedings of the IEEE of OCEANS Conference, p. 6, May 2009.

[32] S. Mason, C. Berger, S. Zhou, K. Ball, L. Freitag, and P. Willett, "An OFDM design for underwater acoustic channels with Doppler spread," in Proceedings of the IEEE Digital Signal Processing Workshop (DSP '09), pp. 138-143, Marco Island, Fla, USA, 2009.

[33] C. R. Berger, S. Zhou, J. C. Preisig, and P. Willett, "Sparse channel estimation for multicarrier underwater acoustic communication: from subspace methods to compressed sensing," IEEE Transactions on Signal Processing, vol. 58, no. 3, pp. 17081721, 2010.

[34] W. Li and J. C. Preisig, "Estimation of rapidly time-varying sparse channels," IEEE Journal of Oceanic Engineering, vol. 32, no. 4, pp. 927-939, 2007.

[35] S. G. Mallat and Z. Zhang, "Matching pursuits with timefrequency dictionaries," IEEE Transactions on Signal Processing, vol. 41, no. 12, pp. 3397-3415, 1993.

[36] J. Bertrand, P. Bertrand, and J. P. Ovarlez, "The Melin transform," in The Transorms and Applications Handbook, A. Poularikas, Ed., 1996.

[37] N. F. Josso, J. J. Zhang, A. Papandreou-Suppappola et al., "Wideband discrete transformation of acoustic signals in underwater environments," in Proceedings of the Asilomar Conference on Signals, Systems, and Computers, pp. 118-122, Pacific Grove, Calif, USA, 2009.

[38] R. A. Altes, "Wide-band, proportional-bandwidth WignerVille analysis," IEEE Transactions on Acoustics, Speech, and Signal Processing, vol. 38, no. 6, pp. 1005-1012, 1990.

[39] A. Papandreou-Suppappola, R. L. Murray, B. G. Iem, and G. F. Boudreaux-Bartels, "Group delay shift covariant quadratic time-frequency representations," IEEE Transactions on Signal Processing, vol. 49, no. 11, pp. 2549-2564, 2001.

[40] A. Papandreou, F. Hlawatsch, and G. F. BoudreauxBartels, "Hyperbolic class of quadratic time-frequency representations-part I: constant-Q warping, the hyperbolic paradigm, properties, and members," IEEE Transactions on Signal Processing, vol. 41, no. 12, pp. 3425-3444, 1993.

[41] R. G. Baraniuk and D. L. Jones, "Unitary equivalence: a new twist on signal processing," IEEE Transactions on Signal Processing, vol. 43, no. 10, pp. 2269-2282, 1995.

[42] A. Jarrot, C. Ioana, and A. Quinquis, "Toward the use of the time warping principle with discrete time sequences," Journal of Computers, vol. 2, pp. 49-55, 2007.

[43] A. Papandreou-Suppappola and S. B. Suppappola, "Analysis and classification of time-varying signals with multiple timefrequency structures," IEEE Signal Processing Letters, vol. 9, no. 3, pp. 92-95, 2002. 
[44] B. Harris and S. Kramer, "Asymptotic evaluation of the ambiguity functions of high-gain FM matched filter sonar systems," Proceedings of the IEEE, vol. 56, pp. 2149-2157, 1968.

[45] S. Kramer, "Doppler and acceleration tolerances of high-gain, wideband linear FM correlation sonars," Proceedings of the IEEE, vol. 55, pp. 627-636, 1967.

[46] W. S. Hodgkiss, H. C. Song, M. Badiey, A. Song, and M. Siderius, "Trip report of the Kauai Acomms MURI 2008 (KAM08) Experiment," July, 2008.

[47] J. P. Ovarlez, Mellin transform: a tool for broadband signal analysis, Ph.D. dissertation, Cergy-Pontoise University, 1992. 